\title{
Reduced Levels of Cerebrospinal Fluid/Plasma A $\beta 40$ as an Early Biomarker for Cerebral Amyloid Angiopathy in RTg-DI Rats
}

\author{
Xiaoyue Zhu ${ }^{1}$, Feng Xu ${ }^{1}$, Michael D. Hoos ${ }^{1,2}{ }^{,}$Hedok Lee $^{3}$, Helene Benveniste ${ }^{3}$ and \\ William E. Van Nostrand ${ }^{1, *(D)}$ \\ 1 George \& Anne Ryan Institute for Neuroscience, Department of Biomedical and Pharmaceutical Sciences, \\ University of Rhode Island, Kingston, RI 02881, USA; xiaoyuezhu123@uri.edu (X.Z.); feng_xu@uri.edu (F.X.); \\ mhoos@enzolifesciences.com (M.D.H.) \\ 2 Enzo Life Sciences, 10 Executive Blvd, Farmingdale, NY 11735, USA \\ 3 Department of Anesthesiology, Yale University, New Haven, CT 06520, USA; hedoklee@gmail.com (H.L.); \\ helene.benveniste@yale.edu (H.B.) \\ * Correspondence: wvannostrand@uri.edu; Tel.: +1-401-874-2363
}

Received: 2 December 2019; Accepted: 30 December 2019; Published: 1 January 2020

check for updates

\begin{abstract}
The accumulation of fibrillar amyloid $\beta$-protein $(A \beta)$ in blood vessels of the brain, the condition known as cerebral amyloid angiopathy (CAA), is a common small vessel disease that promotes cognitive impairment and is strongly associated with Alzheimer's disease. Presently, the clinical diagnosis of this condition relies on neuroimaging markers largely associated with cerebral macro/microbleeds. However, these are markers of late-stage disease detected after extensive cerebral vascular amyloid accumulation has become chronic. Recently, we generated a novel transgenic rat model of CAA (rTg-DI) that recapitulates multiple aspects of human CAA disease with the progressive accumulation of cerebral vascular amyloid, largely composed of $A \beta 40$, and the consistent emergence of subsequent microbleeds. Here, we investigated the levels of A $\beta 40$ in the cerebrospinal fluid (CSF) and plasma of rTg-DI rats as CAA progressed from inception to late stage disease. The levels of $\mathrm{A} \beta 40$ in CSF and plasma precipitously dropped at the early onset of CAA accumulation at three months of age and continued to decrease with the progression of disease. Notably, the reduction in $\mathrm{CSF} /$ plasma $\mathrm{A} \beta 40$ levels preceded the emergence of cerebral microbleeds, which first occurred at about six months of age, as detected by in vivo magnetic resonance imaging and histological staining of brain tissue. These findings support the concept that reduced CSF/plasma levels of A $\beta 40$ could serve as a biomarker for early stage CAA disease prior to the onset of cerebral microbleeds for future therapeutic intervention.
\end{abstract}

Keywords: cerebral amyloid angiopathy; amyloid $\beta$-protein; transgenic rat; cerebrospinal fluid; biomarker

\section{Introduction}

Cerebral amyloid angiopathy (CAA) is a common cerebral small vessel disease that involves the accumulation of amyloid $\beta$-protein $(\mathrm{A} \beta)$ primarily in small- and medium-sized arteries and arterioles of the meninges and cerebral cortex as well as along the capillaries of the cerebral microvasculature [1-4]. CAA is found, to varying degrees, in nearly $80 \%$ of elderly individuals $[5,6]$. Since CAA results from cerebral vascular deposition of $A \beta$, it is not surprising that this condition commonly coexists in patients with Alzheimer's disease (AD) [1,2]. However, independent of AD, clinically CAA is a significant contributor to vascular-mediated cognitive impairment and dementia (VCID) [3,4,7]. As a prominent small vessel disease CAA contributes to the cognitive decline in VCID in several ways 
by promoting perivascular neuroinflammation, impaired cerebral blood flow and ischemic infarcts, cerebral microbleeds, and larger hemorrhages, all of which can result in neuronal dysfunction, neuronal loss and white matter damage [3,8-10].

Presently, the clinical diagnosis of CAA primarily relies on the detection of cerebral microbleeds and larger hemorrhages by imaging techniques [10,11]. Previously, a set of criteria was established, known as the 'Boston Criteria', that determines a diagnosis of probable CAA based on the presence and anatomical location of cerebral microbleeds [11]. An updated version of the "Boston Criteria" was proposed incorporating additional key imaging biomarkers that detect other cerebral vascular injuries associated with amyloid containing vessels including cortical superficial siderosis, enlarged perivascular spaces and white matter hyperintensities [12-14]. Also, anatomical distribution patterns of amyloid accumulation in whole brain detected by positron emission tomography (PET) and A $\beta$ specific radiotracer imaging studies has been informative $[15,16]$. Although these additional imaging markers have improved the clinical diagnosis of probable CAA, they largely represent signs of late-stage disease that emerge after the extensive accumulation of cerebral vascular amyloid has become chronic. Markers that detect the earlier stages of CAA, prior to these late-stage imaging indications, would be beneficial to monitor disease progression in patients while at the same time avoiding therapeutic interventions which might trigger cerebral hemorrhage such as thrombolytic agents $[17,18]$. Alternatively, biomarkers of early onset CAA disease would help to identify patients that could be enrolled in novel therapeutically directed clinical trials.

Recently, we generated a novel transgenic rat model of CAA designated rTg-DI that faithfully recapitulates many features of human capillary CAA type-1 [19]. The rTg-DI rats express human $\mathrm{A} \beta \mathrm{PP}$ in neurons harboring the familial CAA Dutch E693Q/IowaD694N mutations and produce chimeric Dutch/Iowa mutant $A \beta$ in brain. This model exhibits early-onset and progressive microvascular/capillary amyloid accumulation in many forebrain brain regions including the cortex, hippocampus and thalamus that is largely composed of the $\mathrm{A} \beta 40$ peptide. However, there is little accumulation of fibrillar amyloid in larger cerebral vessels in this model. The deposition of cerebral microvascular amyloid is accompanied by robust perivascular neuroinflammation and associated with regular microbleeds [19]. Accordingly, rTg-DI rats provide an invaluable preclinical platform to follow development of CAA pathologies.

In the present study, we show that rTg-DI rats exhibit consistent and progressive accumulation of primarily $A \beta 40$ peptide in the form of microvascular CAA throughout the cortex, hippocampus and thalamus starting at about three months of age. In addition, rTg-DI rats develop consistent cerebral microbleeds that are readily detected by magnetic resonance imaging (MRI) beginning at six months of age with expansion to twelve months of age and confirmed by histological analysis of perivascular hemosiderin deposits at these ages. Measurements of cerebrospinal fluid (CSF) A $\beta 40$ in one month old rTg-DI rats, that do not yet exhibit CAA, represent the homeostatic levels of this peptide prior to disease onset. However, at three months of age, when rTg-DI rats start exhibiting CAA but do not yet show microbleeds, the CSF levels of A $\beta 40$ precipitously and uniformly dropped. At six months of age and later, with further accumulation of CAA and emergence of microbleeds in the brain, CSF levels of $A \beta 40$ continued to drop. Parallel analysis of rTg-DI plasma A $\beta 40$ showed similar trends with lower levels at the onset of CAA at three months and further reductions at six to twelve months. These findings strongly indicate that a reduction in A $\beta 40$ levels in biological fluids represent an early disease related biomarker that correlates with the onset of CAA prior to the emergence of subsequent cerebral microbleeds that are detected by neuroimaging and histological confirmation. Finally, this study underscores the translational utility of rTg-DI rats as a valid preclinical model to further develop biomarkers and a platform to test therapeutic interventions for CAA. 


\section{Results}

\section{1. rTg-DI Rats Show Early-Onset and Progressive Accumulation of Cerebral Vascular Amyloid}

rTg-DI rats were designed to specifically express human A $\beta$ PP harboring the adjacent Dutch E693Q and Iowa D694N familial CAA mutations in neurons in the brain [19]. The accumulation of chimeric Dutch E22Q/Iowa D23N CAA mutant human A $\beta$ in rTg-DI rats over a period of twelve months was measured by ELISA analysis of soluble and insoluble brain fractions (Figure 1). The levels of soluble $A \beta 40$ were very low at one month of age but rose over three to twelve months of age. In contrast, soluble $A \beta 42$ levels were extremely low at one month and remained low through twelve months. On the other hand, the levels of insoluble A $\beta 40$ were relatively low at one month but dramatically increased from three to twelve months. In contrast, the levels of insoluble A $\beta 42$ were $\leq 10 \%$ the levels of insoluble $\mathrm{A} \beta 40$ at each age. These findings indicate that the accumulation of $\mathrm{A} \beta$ peptides in rTg-DI rats is very low at one month, but markedly increases as the rats age to twelve months and is primarily composed of $A \beta 40$.

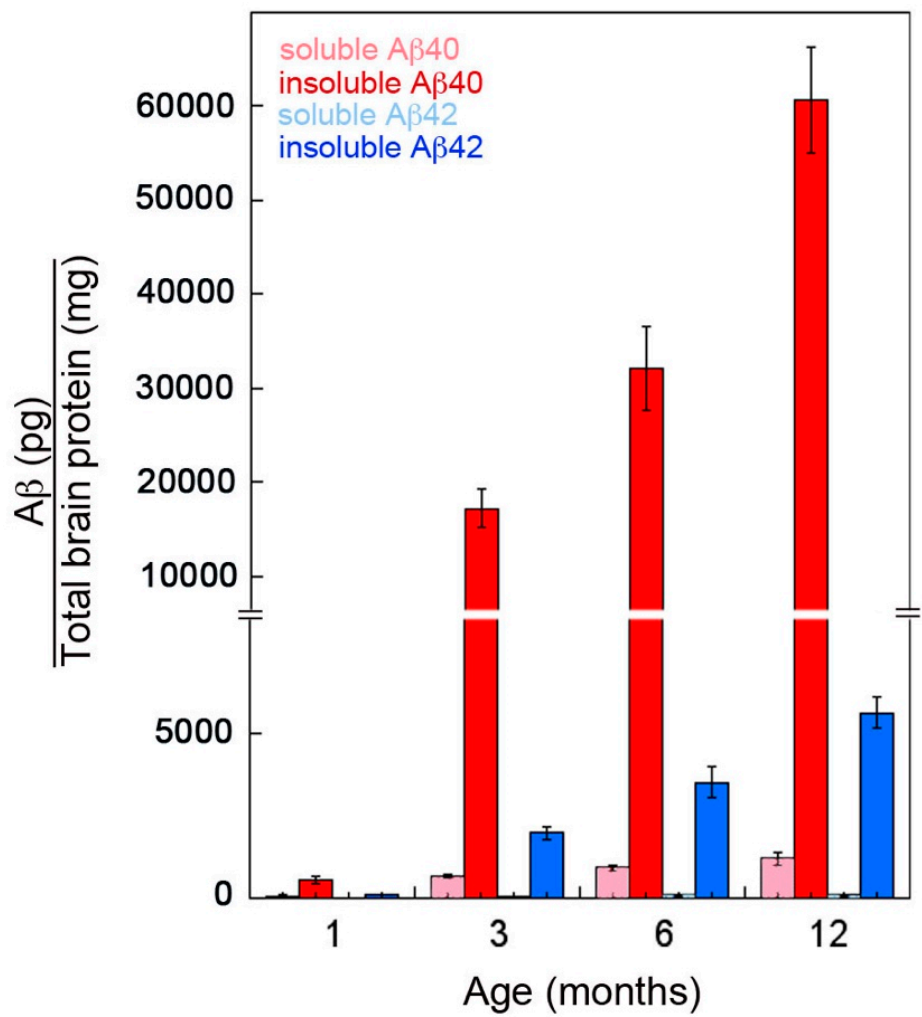

Figure 1. Progressive accumulation of $A \beta$ peptides in rTg-DI rats. The levels of soluble and insoluble $\mathrm{A} \beta 40$ and $\mathrm{A} \beta 42$ peptides in the forebrain of $\mathrm{rTg}$-DI rats aged from one to twelve months were measured by ELISA as described in "Methods". The data presented are the means \pm S.D. of triplicate measurements performed in 5-6 rTg-DI rats per age group.

We recently reported that rTg-DI rats develop the progressive accumulation of cerebral microvascular amyloid that is largely composed of A $\beta 40$ [19]. The ELISA data show low levels of cerebral A $\beta$ peptides at one month (Figure 1). Similarly, at one month of age there was no evidence of microvascular CAA present in rTg-DI rats (Figure 2A). However, from three to twelve months there was the emergence and dramatic increase in the amount of microvascular CAA that paralleled the striking increase of cerebral (soluble and insoluble) A $\beta 40$ peptide (Figure $2 \mathrm{~B}-\mathrm{E}$ ). Together, these findings indicate that elevated cerebral A $\beta 40$ levels and microvascular CAA levels both emerge at about three months of age and dramatically increase over the course of twelve months in rTg-DI rats. 

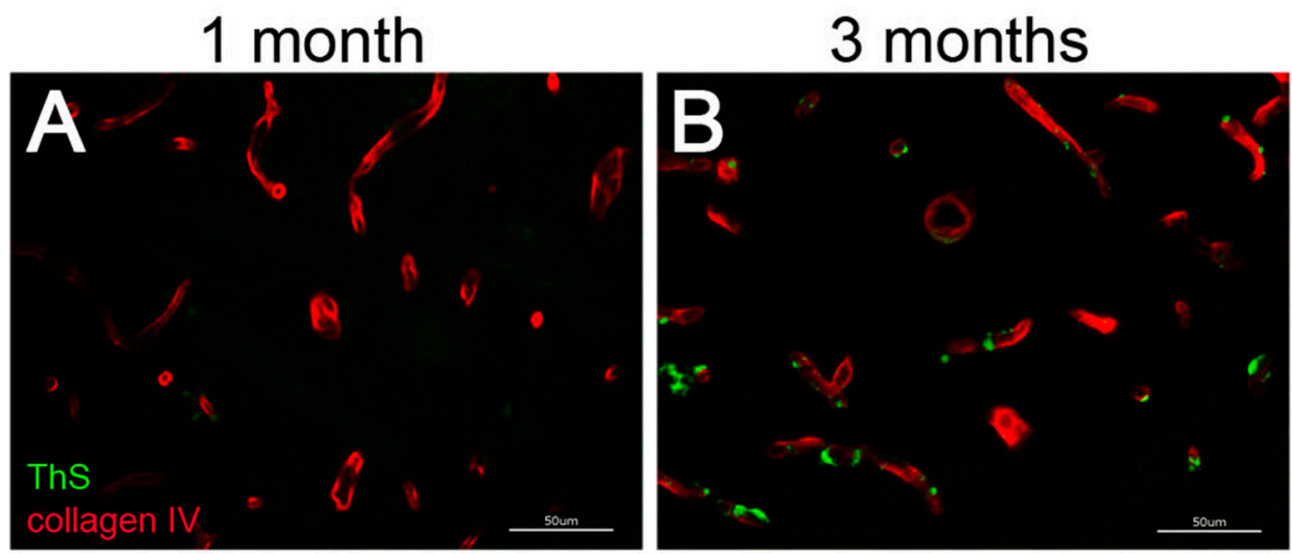

\section{6 months}

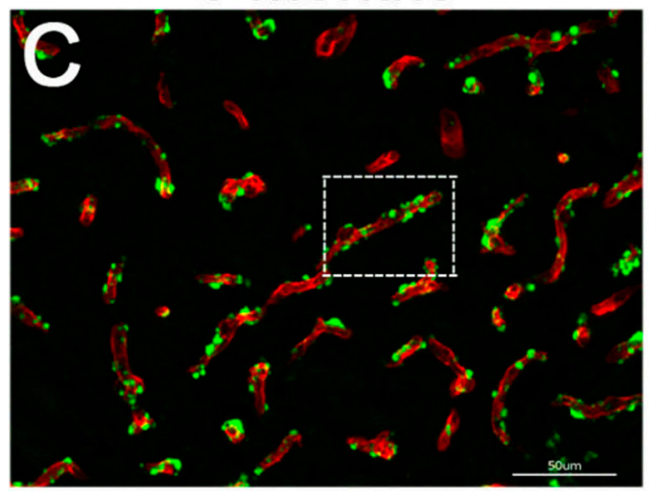

12 months
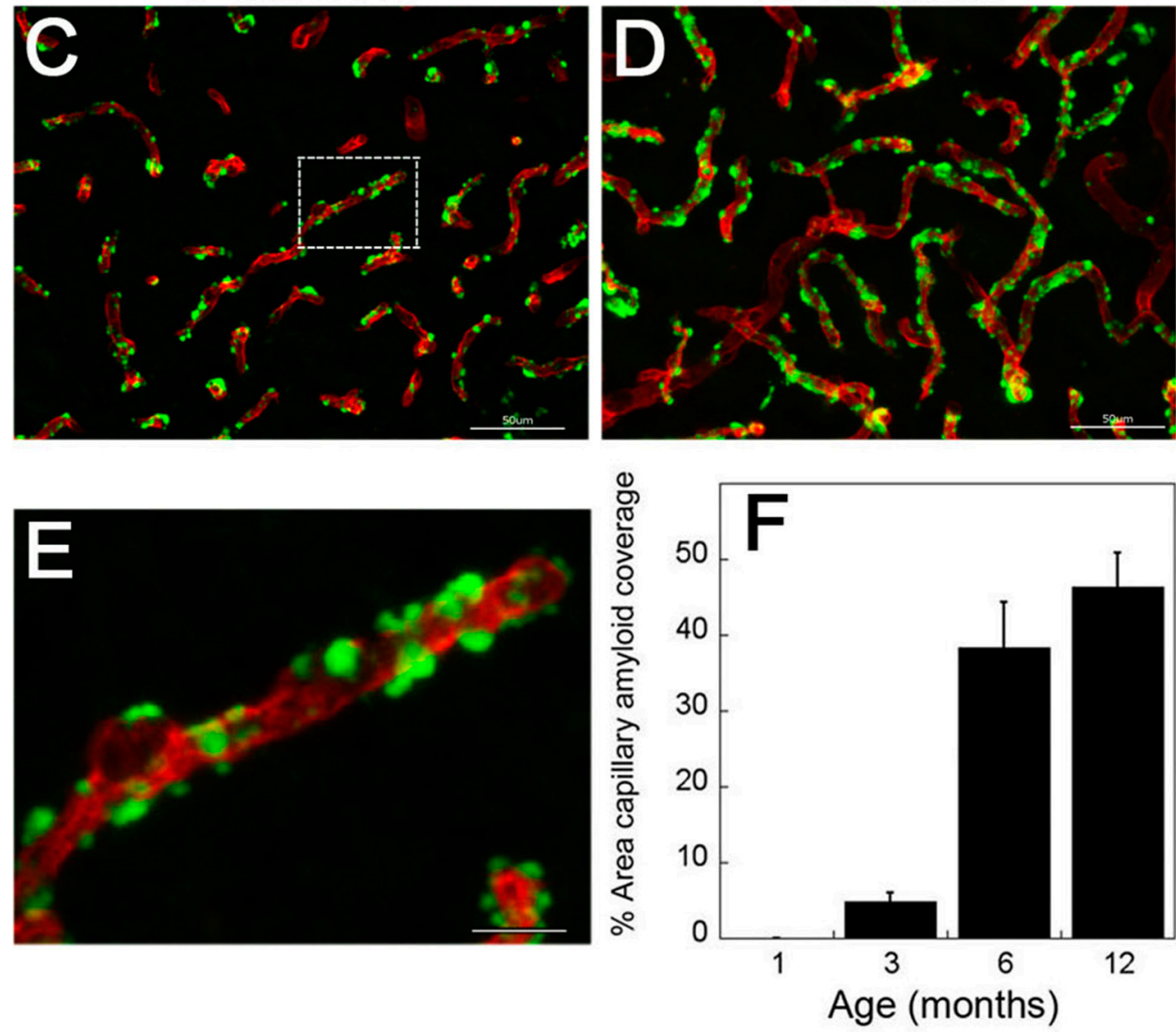

Figure 2. Progressive accumulation of cerebral microvascular amyloid in rTg-DI rats. Representative brain sections showing the thalamic region from rTg-DI rats aged to one month (A), three months (B), six months (C) and twelve months (D) that were stained for fibrillar amyloid using thioflavin-S (green) and immunolabeled for collagen type IV to identify cerebral microvessels (red). Scale bars $=50 \mu \mathrm{M}$. (E) Enlarged inset of panel C showing capillary localization of fibrillar amyloid. Scale bar $=10 \mu \mathrm{m}$. (F) Quantitation of microvascular thioflavin-S positive amyloid load in the thalamic region of rTg-DI rats aged one to twelve months. Data shown are mean \pm S.D. of 5-6 rTg-DI rats per group.

\section{2. rTg-DI Rats Develop Consistent Thalamic Microbleeds Detected by MRI and Histology}

We longitudinally evaluated the emergence of cerebral microbleeds as detected by $\mathrm{T} 2 *$ mapping by MRI from the onset of microvascular CAA at three months to late-stage disease at nine months of age. In rTg-DI rats, the quantitative T2* maps (Figure 3C) clearly displayed bilateral thalamic 'bleeds' 
as represented by distinct areas with very low $\mathrm{T} 2^{*}$ values $\left(\mathrm{T} 2^{*} \leq 20 \mathrm{~ms}\right)$ at nine months (noted by black arrows), consistent with previous findings reported by ex vivo MRI [19]. Notably, wild-type rats showed no evidence of microbleeds over the course of this study (Figure 3B,D). Thalamic microbleeds were consistently detected in the four rTg-DI rats on T2* maps as early as six months of age (Figure 3D,E). The quantitative $\mathrm{T} 2 *$ maps allowed for assessment of microbleed volume changes over time in rTg-DI rats and this analysis revealed emergence of small microbleeds $\left(\sim 2 \mathrm{~mm}^{3}\right)$ at six months of age with up to three-fold volume expansion as the animals aged from six to nine months (Figure 3D,E).
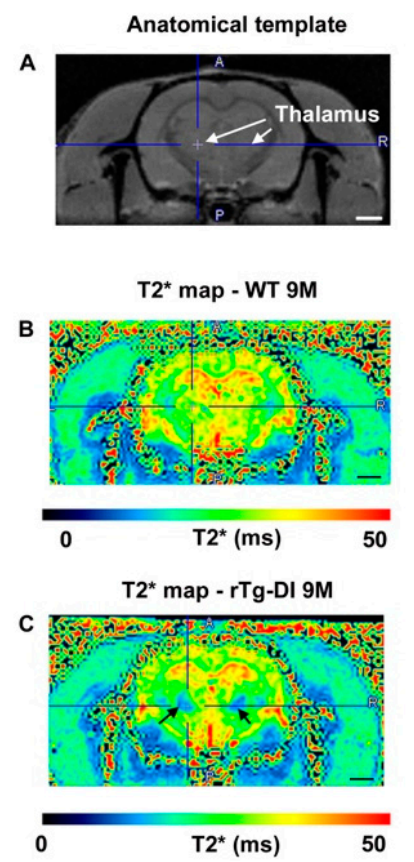
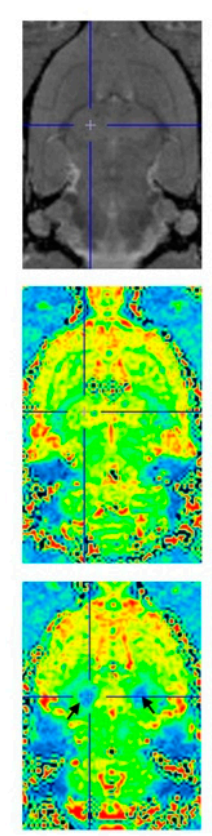
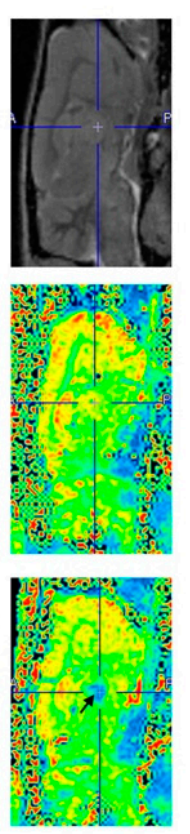

\section{D}

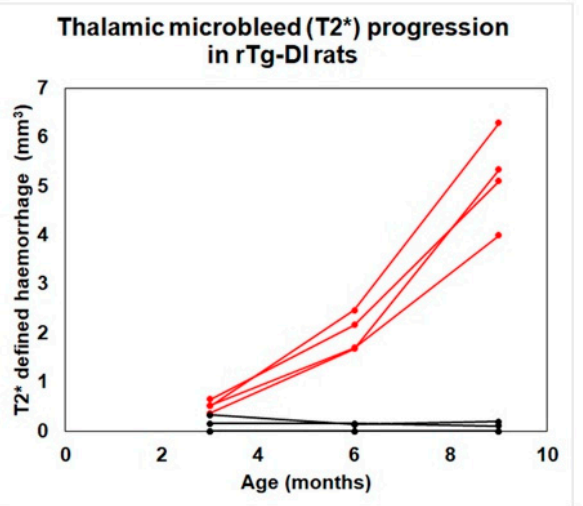

E
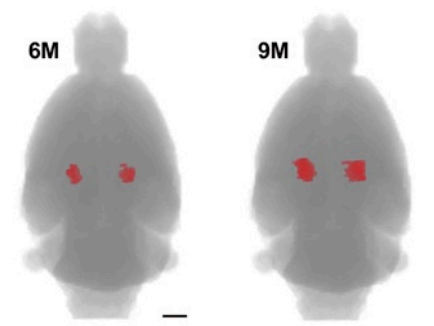

Figure 3. Microbleed progression in thalamus of rTg-DI rats over nine months visualized by MRI. Female rTg-DI CAA rats and wild-type rats were scanned longitudinally at 3, 6 and 9 months of age to track development of microbleeds as defined by T2* parametric mapping in rTg-DI rats. (A) Proton density weighted (PDW) anatomical MRIs presented in three orthogonal planes from a 3-month old rTg-DI CAA for demonstrating the position of the thalamus (white arrows) where the presence of microbleeds is typically noted. (B) T2* parametric, color coded images of the brain presented in three orthogonal planes from a 9 months of age wild type rat. The 'blue' and 'red' colors represent low and high T2* values, respectively. (C) T2* brain map from a $9 \mathrm{M}$ old rTg-DI rat in the same orthogonal planes as in $\mathrm{B}$, with black arrows pointing towards large, dark blue areas in the thalamus representing low $\mathrm{T}^{*}$ $(\leq 20 \mathrm{~ms}$ ) values indicating the presence of ferritin (hemorrhage). Note that the location of the thalamic microbleeds is symmetrical. (D) Quantitative assessment of thalamic microhemorrhage progression over time as defined by T2* $20 \mathrm{~ms}$ from four different rTg-DI CAA rats (red) in comparison to four wild-type rats (black). Small hemorrhages start emerging at 6 months (but not at 3 months) and continue to expand to nine months (on average a three-fold increase in volume). (E) 3D volume rendering of the microbleed in a rTg-DI rat based on $\mathrm{T}^{*} \leq 20 \mathrm{~ms}$, showing the expansion of the microhemorrhage area over time. Scale bars $=3 \mathrm{~mm}$.

To support the MRI findings presented in Figure 3, we performed quantitative histological evaluation for perivascular hemosiderin deposits for further confirmation of thalamic cerebral microbleeds in rTg-DI rats as they aged from one to twelve months. In one month old rTg-DI rats, prior to microvascular CAA deposition, no hemosiderin deposits were detected in the thalamus (Figure 4A). Similarly, at three months of age, when microvascular CAA appears, there is still no histological evidence for cerebral microbleeds (Figure $4 \mathrm{~B}$ ), consistent with the MR imaging data presented in Figure 3. However, at six months of age, with more extensive CAA, thalamic perivascular 
hemosiderin deposits are evident (Figure 4C,E). The extent of hemosiderin deposition increases sharply at twelve months of age (Figure 4D,E), again highly consistent with the imaging findings. Together, these findings clearly show that cerebral microbleeds do not develop in rTg-DI rats until several months after the onset of cerebral microvascular amyloid deposition.

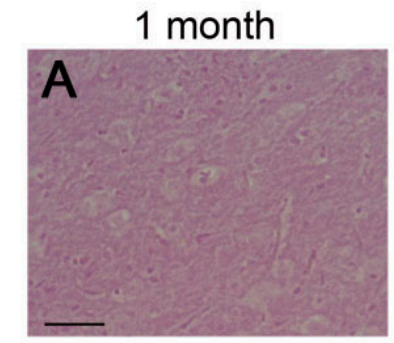

6 months

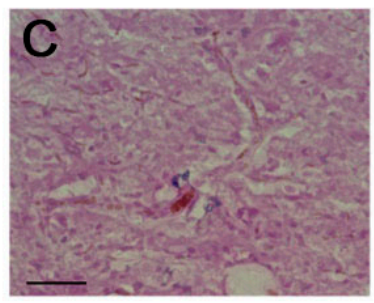

3 months

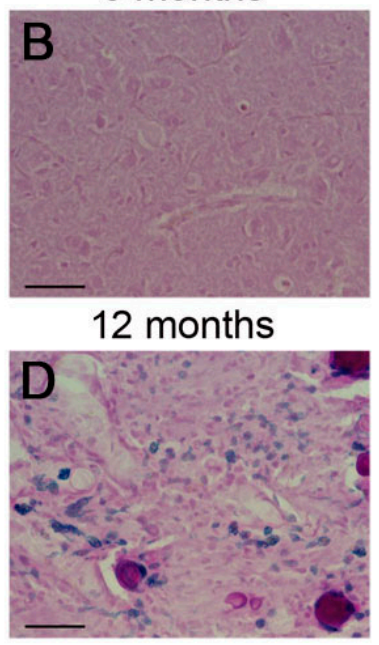

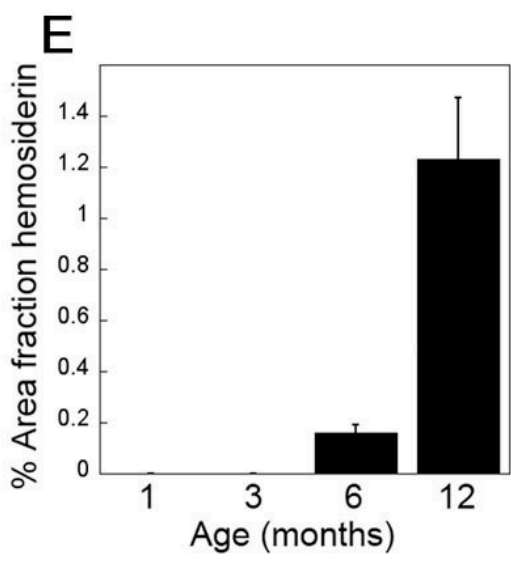

Figure 4. Emergence of cerebral microbleeds in rTg-DI rats. Representative brain sections showing the thalamic region from $\mathrm{rTg}$-DI rats aged to one month (A), three months (B), six months (C) and twelve months (D) that were stained for hemosiderin to identify microhemorrhages (blue). Scale bars $=50 \mu \mathrm{m}$. (E) The percent area fraction of hemosiderin staining was quantitated in the thalamus of 1, 3, 6, and 12 months old rTg-DI rats. Data represent the mean \pm S.D. of 6-7 rTg-DI rats per group.

\subsection{CSF and Plasma Levels of A 40 Markedly Drop at the Inception of Microvascular CAA in rTg-DI Rats}

Previously, it was reported that the levels of $A \beta 40$, the major isoform of $A \beta$ found in CAA deposits, are reduced in probable CAA patients diagnosed by the presence of cerebral microbleeds [20-22]. Therefore, we performed cross sectional measurements of A $\beta 40$ in the CSF of cohorts of rTg-DI rats as they progressed through different stages of disease to determine the trajectory of this marker. At one month, prior to the accumulation of appreciable cerebral $A \beta 40$ or evidence of any microvascular CAA, the mean CSF levels of $A \beta 40$ were $\approx 5500 \mathrm{pg} / \mathrm{mL}$ (Figure 5). At three months, with the marked accumulation of cerebral $\mathrm{A} \beta 40$ and the initial formation of $\mathrm{CAA}$, there was a precipitous $\approx 70 \%$ drop in CSF A $\beta 40$ levels to $\approx 1750 \mathrm{pg} / \mathrm{mL}(p<0.0001)$. As rTg-DI rats aged further to six and twelve months, with more extensive cerebral $\mathrm{A} \beta 40$ and CAA accumulation, coupled with the emergence and progression of cerebral microbleeds, there were further declines in CSF A $\beta 40$ levels to $\approx 18 \%$ and $\approx 10 \%$ the initial levels observed in one month old rTg-DI rats. Parallel measurements of CSF A $\beta 42$ levels, although much lower than $A \beta 40$ levels, showed similar declines with the onset and progression of microvascular CAA (data not shown).

We next extended the ELISA analysis for $A \beta 40$ to plasma samples collected from the different aged rTg-DI rats (Figure 6). At one month, prior to the onset of microvascular CAA, the plasma levels of $A \beta 40$ were $\approx 60$-fold lower than what was measured in CSF and tended to show somewhat more variation. Nevertheless, at three months, with the onset of microvascular CAA, there was a significant $\approx 54 \%$ drop in the plasma levels of $\mathrm{A} \beta 40(p<0.02)$. The levels of plasma A $\beta 40$ continued to drop at six months of age to $\approx 12 \%$ of the initial one-month plasma levels and, in this case, appeared to level off to twelve months of age. A $\beta 42$ levels in plasma appear to be extremely low and were not detected in the ELISA. Together, these findings show that both CSF and plasma levels of A $\beta 40$ in rTg-DI rats are relatively high prior to the onset of CAA but sharply decline when cerebral microvascular amyloid begins to deposit. This acute decrease in CSF and plasma A $\beta 40$ occurred early and precedes the 
emergence of cerebral microbleeds, but continues to decline as the animals age further and the disease severity progresses.

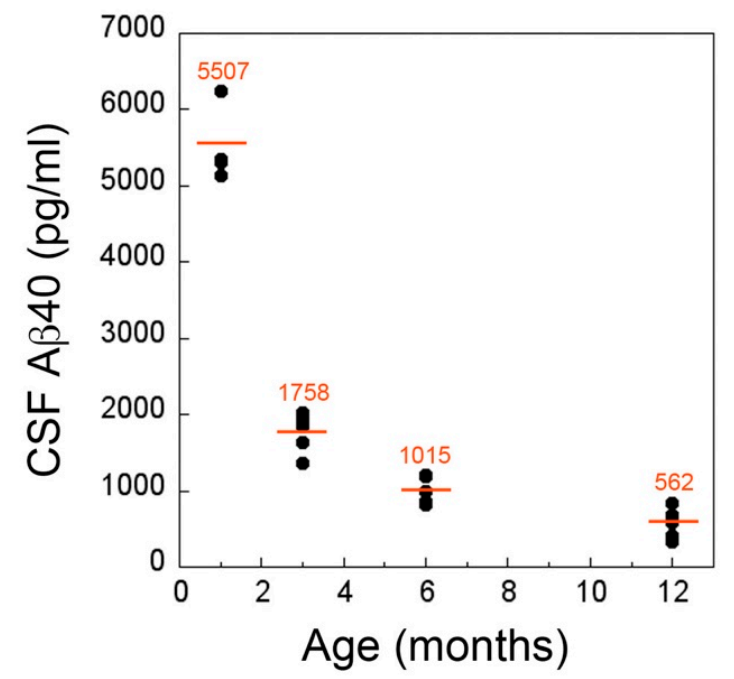

Figure 5. Decreased levels of $A \beta 40$ in CSF of rTg-DI rats at the inception and progression of microvascular CAA. The levels of A $\beta 40$ in CSF collected from rTg-DI rats as they aged from one to twelve months was determined by ELISA. The data show the mean CSF A $\beta 40$ levels at each time point from $n=5-6 \mathrm{rTg}$-DI rats per group. At three months of age, when CAA first appears, there is precipitous drop in CSF A $\beta 40$ levels that continue to decline with progressing CAA.

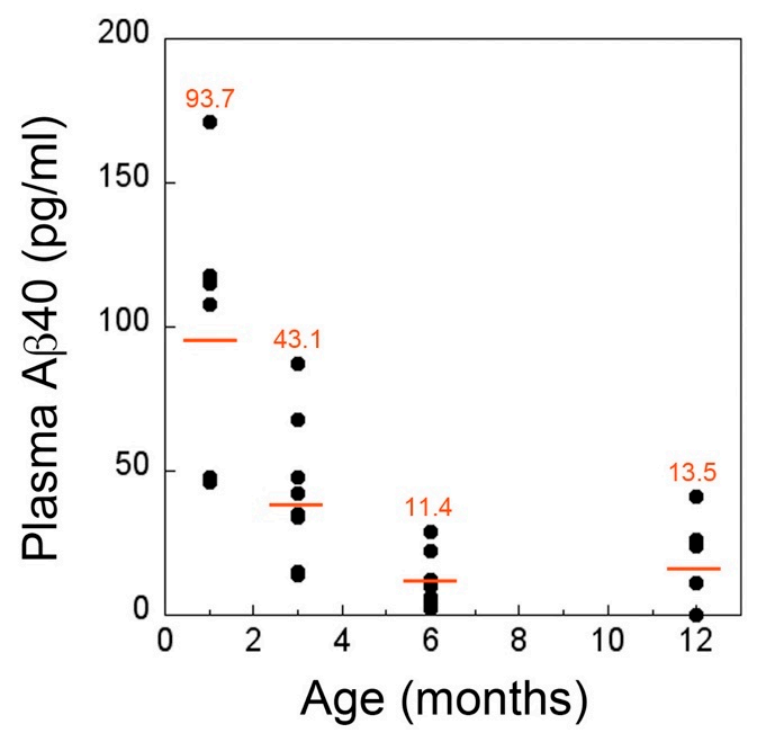

Figure 6. Decreased levels of $A \beta 40$ in plasma of $\mathrm{rTg}$-DI rats at the inception and progression of microvascular CAA. The levels of A $\beta 40$ in plasma collected from rTg-DI rats as they aged from one to twelve months was determined by ELISA. The data show the mean plasma A $\beta 40$ levels at each time point from $n=6-8 \mathrm{rTg}$-DI rats per group. At three months of age, when CAA first appears, there is marked reduction in plasma $\mathrm{A} \beta 40$ levels that continue to decline with progressing CAA.

\section{Discussion}

CAA is a common cerebral small vessel disease of the elderly and a prominent comorbidity of AD that promotes and exacerbates VCID, yet our ability to diagnose CAA remains limited to late-stage neuroimaging markers for this condition. The neuroimaging markers used in the 'Boston Criteria' diagnosis is centered around vascular and perivascular changes that are associated with cerebral blood vessels impacted by the chronic and extensive accumulation of cerebral vascular amyloid. The 
major scoring factors for clinical diagnosis of CAA is the lobar presence of a large macrobleed, which occurs in very severe cases of disease, or the presence of multiple lobar microbleeds, which is more common and observed in chronic, late-stage disease with extensive cerebral vascular amyloid [11]. Additional neuroimaging markers that aid clinical diagnosis include perivascular changes that occur in the vicinity around CAA-affected vessels. For example, cortical superficial siderosis indicates perivascular iron accumulation in pial arteries and arterioles reflecting cerebral microbleeds $[12,14,23]$. Another noted perivascular change associated with CAA is the presence of dilated perivascular spaces, particularly around penetrating arterioles with amyloid deposition [8,24]. Although the cause of this perivascular alteration remains unclear this space contains CSF and interstitial fluid (ISF) that under normal conditions plays an important role in A $\beta$ clearance from brain [25-28]. The presence of amyloid in these vessels promotes local perivascular inflammation and may disrupt normal CSF flow and exchange with ISF leading to enlargement of these spaces [4,29]. Although these neuroimaging markers are useful in diagnosing chronic, late-stage disease with extensive CAA, they are not helpful in detecting early stages of emerging cerebral vascular amyloid accumulation that could be more amenable to therapeutic interventional strategies and allow for clearance of the emerging perivascular A $\beta$ deposits.

Parenchymal amyloid plaques commonly found in AD primarily contain the longer A $\beta 42$ species. Previous studies have shown that as brain parenchymal plaques accumulate in AD there is a significant decrease in the levels of A $\beta 42$ detected in CSF as this peptide accumulates in brain [30,31]. Accordingly, CSF $A \beta 42$ levels have provided a surrogate biomarker for the parenchymal plaque burden in brain and have assisted in the clinical diagnosis of $\mathrm{AD}[32,33]$. In contrast to plaques, CAA deposits are largely composed of the shorter $A \beta 40$ peptide [1,2]. This suggests that a decrease in CSF A $\beta 40$ levels could reflect the presence and burden of CAA. Indeed, studies have shown that patients diagnosed with CAA, based on late-stage neuroimaging biomarkers for cerebral microbleeds and cortical superficial siderosis, presented with lower levels of CSF A $\beta 40$ [20,34]. However, the utility of CSF A $\beta 40$ levels to serve as a potential biomarker for earlier stage CAA, prior to the onset of microbleeds and other perivascular changes, remains unclear.

Most animal models to study CAA have largely involved the use of transgenic mouse lines that express human A $\beta P P$ generally producing highly elevated levels of $A \beta$ peptides in brain with or without familial CAA mutations [35-38]. Although these mouse models have been helpful in studying the pathogenesis of CAA their usefulness in modeling the human disease has been met with limitations including the small size of the mouse brain, which has hampered neuroimaging studies, and the variable presentation of cerebral microbleeds. Our recently generated transgenic rat model rTg-DI more faithfully recapitulates many of the pathological features of human small vessel CAA including early-onset and progressive accumulation of cerebral microvascular fibrillar amyloid, perivascular neuroinflammation, consistent and robust development of cerebral microhemorrhages and small vessel occlusions and behavioral deficits [19]. The rTg-DI rats used in this study provided a unique opportunity to evaluate the trajectories of A $\beta 40$ in CSF and plasma in cohorts of animals that consistently progressed from the early-onset to late-stage disease pathologies. Indeed, from the accumulation of $A \beta$ peptides in brain, to the development of $C A A$, to the emergence and expansion of microbleeds and to measurements of CSF/plasma A $\beta 40$ the cohorts rTg-DI rats progressed through each stage of disease with uniformity.

The CSF compartment is an important exchange reservoir with the ISF compartment that surrounds the cellular components of the brain. This dynamic interaction plays an important clearance route for $\mathrm{A} \beta$ and other cellular metabolic waste products through glymphatic system transport and/or alternate intramural perivascular drainage pathways [25-28]. Thus, the CSF compartment can reflect the ongoing clearance of $A \beta$ and provide a snapshot of $A \beta$ pathology and burden in the brain. For example, the chronic pathological accumulation of $A \beta 42$ in parenchymal plaques in AD brain is reflected by decreased levels of this specific $A \beta$ isoform in CSF and is associated with a decline in 
cognitive function [32,33]. Similarly, our findings demonstrate that the selective accumulation of $A \beta 40$ in CAA deposits is reflected by early onset decreased levels of this A $\beta$ species in CSF.

Another important route for $A \beta$ clearance from the CNS is across the blood-brain barrier of the cerebral endothelium into blood for peripheral removal $[39,40]$. This clearance route is facilitated by endothelial $A \beta$ transporters including low density lipoprotein receptor related protein 1 (LRP1) and p-glycoprotein $[39,41,42]$. However, the levels of $A \beta$ peptides in plasma are much lower than in CSF and can be more variable and, therefore, is less reliable in serving as a biomarker for AD [43,44]. In the case of using rTg-DI rats these shortcomings are further compounded by the finding that compared with non-mutated wild-type $A \beta$ the chimeric Dutch/Iowa CAA mutant A $\beta$ poorly binds LRP1 and is much less effectively transported across the blood-brain barrier into the circulation [45]. Indeed, the level of $\mathrm{A} \beta 40$ detected in plasma of rTg-DI rats was $<2 \%$ of what was measured in the CSF. Nevertheless, we still found that A $\beta 40$ levels in plasma of rTg-DI rats dramatically drop with the onset of cerebral microvascular amyloid accumulation, thus mirroring what was observed in the CSF.

We recently reported that in CAA deposits in transgenic mice and humans, as well as in rTg-DI rats, the amyloid fibrils adopt a distinct anti-parallel configuration $[19,46]$. The present findings suggest that once $\mathrm{A} \beta 40$ forms fibrillar amyloid deposits in cerebral microvessels and capillaries, this may act as a nidus for further seeding of additional soluble $A \beta 40$ to expand vascular fibrillar amyloid with this distinct anti-parallel conformation and further impair its clearance through either the CSF or into the peripheral circulation.

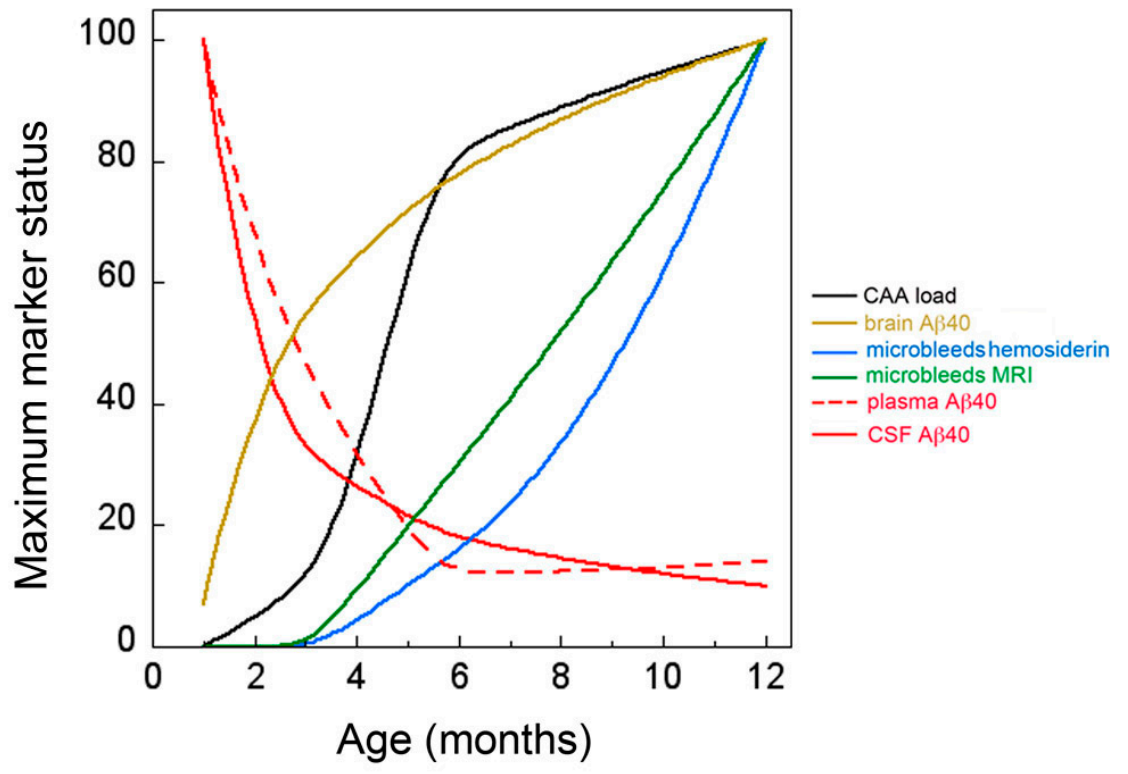

Figure 7. Trajectory of CSF and plasma A $\beta 40$ levels with the progression of CAA and associated microbleed pathology in rTg-DI rats. At one month of age, the levels of A $\beta 40$ in CSF and plasma are relatively high, whereas little $\mathrm{A} \beta 40$ has accumulated in brain tissue, and CAA and microbleeds are not present. At three months of age, the levels of $A \beta 40$ in brain sharply increase and CAA begins to develop. At this same time, there is dramatic decrease in the levels of $A \beta 40$ in both CSF and plasma. At six months of age, the levels of $A \beta 40$ in brain and CAA continue to increase and microbleeds begin to emerge. The levels of $A \beta 40$ in both CSF and plasma continue to decline. Finally, at twelve months of age, there is a continued increase in brain $\mathrm{A} \beta 40$, CAA load and expansion of cerebral microbleeds that is accompanied by further reduction in CSF A $\beta 40$.

The trajectory of disease markers in the rTg-DI model of microvascular CAA are summarized in Figure 7. In rTg-DI rats the accumulation of A $\beta$ peptides in brain and the onset of CAA began early, at about two to three months of age, and dramatically increased over twelve months. The emergence of cerebral microbleeds, confirmed by in vivo MR imaging and histological evaluation, occur significantly 
later starting at around six months, with further expansion at nine and twelve months. The present findings show that at the onset of CAA, much earlier than the emergence of cerebral microbleeds, there is a precipitous drop in the CSF/plasma levels of $A \beta 40$, the chief component of CAA deposits. The reductions in CSF A $\beta 40$ levels in rTg-DI rats are consistent with prior studies using CSF collected from probable CAA patients with MRI confirmed cerebral microbleeds [20-22,34]. However, the present findings show that reductions in CSF/plasma $\mathrm{A} \beta 40$ occur much earlier with the onset of CAA and prior to microbleeds. This underscores the value of this novel model to identify other possible biomarkers that correlate with disease state.

\section{Materials and Methods}

\section{1. $r T g$-DI Rats}

All animal experiments were approved by the local Institutional Animal Care and Use Committees at the University of Rhode Island (project \#AN1718-008; approval dates 12/11/2017-12/10/2020) and Yale University (project \#2019-20132; approval dates 11/1/2019-10/31/2022) and conducted in accordance with the United States Public Health Service's Policy on Humane Care and Use of Laboratory Animals. rTg-DI rats were designed to express human A $\beta P P$ (isoform 770) harboring the Swedish K670N/M671L, Dutch E693Q, and Iowa D694N mutations in neurons under control of the Thy1.2 promoter and produce chimeric Dutch/Iowa CAA mutant A $\beta$ in their brains [19]. Transgenic offspring were determined by PCR analysis of tail DNA. All subsequent analyses were performed with heterozygous transgenic rats.

\subsection{CSF Collection}

CSF was collected from the cisterna magna of rTg-DI rats at designated ages. Rats were deeply anesthetized with inhalation of isoflurane and then mounted on a stereotaxic unit. A midline incision was made beginning between the ear and ending approximately $2.5 \mathrm{~cm}$ caudally. The fascia was retracted and muscles dissected, exposing the atlanto-occipital membrane. Using a \#11 scalpel, a small slit was made along the midline of the membrane and underlying dura under a surgical microscope. The CSF was collected through the dura slit by using a fine tip pipette and aliquoted into sterile Eppendorf tube and frozen at $-80^{\circ} \mathrm{C}$. Approximately $150 \mu \mathrm{L}$ of CSF was collected from each rat.

\subsection{Plasma Isolation}

Rat blood was collected by terminal cardiac puncture from anesthetized rTg-DI rats at the designated ages. Blood was collected in one tenth volume of $3.8 \%$ sodium citrate to prevent coagulation. Blood was centrifuged at $8000 \times g$ for $5 \mathrm{~min}$ at room temperature to remove platelets and cellular components. Plasma samples were stored at $-80^{\circ} \mathrm{C}$ until ELISA analysis.

\subsection{Brain Tissue Collection and Preparation}

Rats were euthanized at designated time points and perfused with cold-PBS, forebrains were removed and dissected through the mid-sagittal plane. One hemisphere was immersion-fixed with $70 \%$ ethanol overnight and subjected to increasing sequential dehydration in ethanol, followed by xylene treatment and embedding in paraffin. Alternatively, brains were fixed with $4 \%$ paraformaldehyde overnight at $4{ }^{\circ} \mathrm{C}$ and subjected to increasing concentrations (10\%, 20\%,30\%) of sucrose in PBS, then embedded in OCT compound (Sakura Finetek Inc., Torrance, CA, USA) and snap-frozen in dry ice. Other hemispheres were collected, frozen on dry ice and stored at $-80^{\circ} \mathrm{C}$. Sagittal sections were cut at $10 \mu \mathrm{m}$ thickness using a Leica RM2135 microtome (Leica Microsystems Inc., Bannockburn, IL, USA), placed in a flotation water bath at $40{ }^{\circ} \mathrm{C}$, and then mounted on Colorfrost/Plus slides (ThermoFisher Scientific, Houston, TX, USA). In some cases, coronal sections were cut at $20 \mu \mathrm{m}$ thickness from frozen brains using a Leica CM1900 cryostat (Leica Microsystems Inc.), stored in PBS with $0.02 \%$ sodium azide at $4{ }^{\circ} \mathrm{C}$. 


\subsection{ELISA Quantitation of A $\beta$ Peptides}

The levels of soluble and insoluble $A \beta 40$ and $A \beta 42$ were determined by performing specific ELISAs as described $[47,48]$. Briefly, brain hemispheres that were flash frozen and pulverized in liquid nitrogen. A soluble fraction was obtained by homogenizing tissue with $10 \mu \mathrm{L} / \mathrm{mg}$ of $1 \mathrm{M}$ sodium carbonate, $500 \mathrm{mM} \mathrm{NaCl}, \mathrm{pH} 11.5$ and $0.5 \mathrm{~mm}$ zirconium oxide beads in a bullet blender. Aliquots were spun at $1600 \times g$ at $4{ }^{\circ} \mathrm{C}$ for $20 \mathrm{~min}$. The supernatant was removed, which was the soluble fraction. The remaining pellet was suspended in $5 \mathrm{M}$ guanidine- $\mathrm{HCl}, 50 \mathrm{mM}$ Tris, $\mathrm{pH} 8.0$ and rotated at room temperature for $3 \mathrm{~h}$. Samples were centrifuged and the supernatant was collected, which was the insoluble fraction. For each of the two fractions, a sandwich ELISA was performed. Antibody reagents for the A $\beta$ ELISAs were generously provided by Lilly Research Laboratories, Indianapolis, IN, USA. In the sandwich ELISAs $A \beta 40$ and $A \beta 42$ peptides were captured using the carboxyl-terminal specific antibodies $\mathrm{m} 2 \mathrm{G} 3$ and $\mathrm{m} 21 \mathrm{~F} 12$, respectively, and biotinylated m3D6, specific for the N-terminus of human A $\beta$, was used for detection followed by streptavidin-HRP (Amdex RPN4401V; Fisher Scientific, Pittsburgh, PA, USA). Plates were developed using KPL SureBlue (SeraCare, Milford, MA, USA) and read using a Spectramax M2 plate reader (Molecular Devices, Sunnyvale, CA, USA). Each sample lysate was measured in triplicate and compared to linear standard curves generated with known concentrations of human A $\beta$. The same ELISA format was used to measure soluble A $\beta$ levels in CSF and plasma collected from each rat.

\subsection{Immunohistochemical Analysis}

Antigen retrieval was performed by treating the tissue sections with proteinase $\mathrm{K}(0.2 \mathrm{mg} / \mathrm{mL})$ for $10 \mathrm{~min}$ at $22^{\circ} \mathrm{C}$. Primary rabbit polyclonal antibody to collagen type IV was used to visualize cerebral microvessels (1:100; ThermoFisher, Rockford, IL, USA). Primary antibody was detected with Alexa Fluor 594-conjugated donkey anti-rabbit secondary antibody (1:1000). Staining for fibrillar amyloid was performed using thioflavin S. Prussian blue iron staining was performed to detect hemosiderin deposits reflecting signs of previous microhemorrhage.

\subsection{Quantitative Histological Analysis of CAA Load and Microbleeds}

The percent area amyloid coverage of cerebral microvessels and percent area iron staining in the thalamic region was determined in rats at each of the specified ages using stereological principles as previously described $[19,36]$.

\subsection{Magnetic Resonance Imaging Analysis}

For non-invasive MRI imaging, rTg-DI rats $(N=4)$ and age-matched WT rats $(N=4)$ were lightly anesthetized with dexmedetomidine $(0.015 \mathrm{mg} / \mathrm{kg} / \mathrm{h})$ and low dose isoflurane $0.5-1 \%$ as previously described [49]. MRI imaging was performed on a Bruker 9.4T MRI and images of the rat brain were acquired using a $40 \mathrm{~mm}$ volume transmit and receive coil. The 3D gradient echo imaging parameters were acquired with the following parameters: TR/TE/FA $=60 \mathrm{~ms} / 2 \sim 32 / 15^{\circ}, \mathrm{NEX}=6$, resolution= $0.23 \times 0.23 \times 0.23 \mathrm{~mm}$, scan time $=50 \mathrm{~min}$. A proton density weighted anatomical MRI of each rat's brain was acquired at the same spatial resolution. Following MRI, the anesthesia was discontinued, and the rats allowed to recover. Rats were scanned at three, six, and nine months of age.

Quantitative 3D T2* maps were calculated from the 3D multiple gradient echo (MGE TE $=2 \sim 32 \mathrm{~ms}$ ) MRIs by assuming mono-exponential relationship between the signal and TEs [50]. Deposition of paramagnetic containing blood product, such as ferritin, and $\mathrm{T} 2^{*}$ values have been reported to be linearly correlated. $\mathrm{T} 2 *$ values $\leq 20 \mathrm{~ms}$ within the thalamus was identified on the parametric $\mathrm{T}^{*}$ maps in each rat using the Amira software segmentation editor (Amira 6.4, ThermoFisher Scientific, Houston, TX, USA). The number of voxel with $\mathrm{T}^{*} \leq 20 \mathrm{~ms}$ was converted into $\mathrm{mm}^{3}$ and used as an estimate of 'total hemorrhagic load' in the thalamus. 


\subsection{Statistical Analysis}

Histological and biochemical data were analyzed by $t$-test at the 0.05 significance level.

Author Contributions: X.Z., F.X., M.D.H., and H.L. performed the experiments; X.Z., F.X., H.L., H.B., and W.E.V.N. contributed to the conception and analysis of the experiments; X.Z., H.B., and W.E.V.N. were major contributors to writing and editing the manuscript. All authors have read and agreed to the published version of the manuscript.

Funding: This research was funded by NIH grants NS094201 (WVN), AG053991 (HB and WVN) and BIONIC project (nr. 733050822), which has been made possible by ZonMW. The BIONIC project is part of 'Memorabel', the research and innovation program for dementia, as part of the Dutch national 'Deltaplan for Dementia': zonmw.nl/dementiaresearch". The BIONIC project is a consortium of Radboudumc, LUMC, ADX Neurosciences, and University of Rhode Island.

Acknowledgments: Antibody reagents for the A $\beta$ ELISAs were generously provided by Lilly Research Laboratories, Indianapolis, IN, USA.

Conflicts of Interest: The authors declare no conflicts of interests.

\section{Abbreviations}

$\begin{array}{ll}\text { A } \beta & \text { Amyloid- } \beta \text { protein } \\ \text { A } \beta \text { PP } & \text { A } \beta \text { precursor protein } \\ \text { AD } & \text { Alzheimer's disease } \\ \text { CAA } & \text { Cerebral amyloid angiopathy } \\ \text { CNS } & \text { Central nervous system } \\ \text { CSF } & \text { Cerebral spinal fluid } \\ \text { ELISA } & \text { Enzyme linked immunosorbent assay } \\ \text { ISF } & \text { Interstitial fluid } \\ \text { LRP1 } & \text { low density lipoprotein receptor related protein } 1 \\ \text { MRI } & \text { Magnetic resonance imaging } \\ \text { rTg-DI } & \text { Transgenic rats expressing Dutch/Iowa mutant A } \beta P P \\ \text { VCID } & \text { Vascular mediated cognitive impairment and dementia }\end{array}$

\section{References}

1. Rensink, A.A.; De Waal, R.M.; Kremer, B.; Verbeek, M.M. Pathogenesis of cerebral amyloid angiopathy. Brain Res. Brain Res. Rev. 2003, 43, 207-223. [CrossRef] [PubMed]

2. Attems, J.; Jellinger, K.; Thal, D.R.; Van Nostrand, W. Review: Sporadic cerebral amyloid angiopathy. Neuropathol. Appl. Neurobiol. 2011, 37, 75-93. [CrossRef] [PubMed]

3. Auriel, E.; Greenberg, S.M. The pathophysiology and clinical presentation of cerebral amyloid angiopathy. Curr. Atheroscler. Rep. 2012, 14, 343-350. [CrossRef] [PubMed]

4. Banerjee, G.; Carare, R.; Cordonnier, C.; Greenberg, S.M.; Schneider, J.A.; Smith, E.E.; Buchem, M.V.; Grond, J.V.; Verbeek, M.M.; Werring, D.J. The increasing impact of cerebral amyloid angiopathy: Essential new insights for clinical practice. J. Neurol. Neurosurg. Psychiatry 2017, 88, 982-994. [CrossRef]

5. Arvanitakis, Z.; Leurgans, S.E.; Wang, Z.; Wilson, R.S.; Bennett, D.A.; Schneider, J.A. Cerebral amyloid angiopathy pathology and cognitive domains in older persons. Ann. Neurol. 2011, 69, 320-327. [CrossRef]

6. Boyle, P.A.; Yu, L.; Nag, S.; Leurgans, S.; Wilson, R.S.; Bennett, D.A.; Schneider, J.A. Cerebral amyloid angiopathy and cognitive outcomes in community-based older persons. Neurology 2015, 85, 1930-1936. [CrossRef]

7. Greenberg, S.M.; Gurol, M.E.; Rosand, J.; Smith, E.E. Amyloid angiopathy-related vascular cognitive impairment. Stroke 2004, 35, 2616-2619. [CrossRef]

8. Martinez-Ramirez, S.; van Rooden, S.; Charidimou, A.; van Opstal, A.M.; Wermer, M.; Gurol, M.E.; Terwindt, G.; van der Grond, J.; Greenberg, S.M.; van Buchem, M.; et al. Perivascular Spaces Volume in Sporadic and Hereditary (Dutch-Type) Cerebral Amyloid Angiopathy. Stroke 2018, 49, 1913-1919. [CrossRef]

9. Xiong, L.; van Veluw, S.J.; Bounemia, N.; Charidimou, A.; Pasi, M.; Boulouis, G.; Reijmer, Y.D.; Giese, A.K.; Davidsdottir, S.; Fotiadis, P.; et al. Cerebral Cortical Microinfarcts on Magnetic Resonance Imaging and Their Association with Cognition in Cerebral Amyloid Angiopathy. Stroke 2018, 49, 2330-2336. [CrossRef] 
10. Viswanathan, A.; Greenberg, S.M. Cerebral amyloid angiopathy in the elderly. Ann. Neurol. 2011, 70, 871-880. [CrossRef]

11. Knudsen, K.A.; Rosand, J.; Karluk, D.; Greenberg, S.M. Clinical diagnosis of cerebral amyloid angiopathy: Validation of the Boston criteria. Neurology 2001, 56, 537-539. [CrossRef] [PubMed]

12. Greenberg, S.M.; Charidimou, A. Diagnosis of Cerebral Amyloid Angiopathy: Evolution of the Boston Criteria. Stroke 2018, 49, 491-497. [CrossRef] [PubMed]

13. Charidimou, A.; Frosch, M.P.; Salman, R.A.; Baron, J.C.; Cordonnier, C.; Hernandez-Guillamon, M.; Linn, J.; Raposo, N.; Rodrigues, M.; Romero, J.R.; et al. Advancing diagnostic criteria for sporadic cerebral amyloid angiopathy: Study protocol for a multicenter MRI-pathology validation of Boston criteria v2.0. Int. J. Stroke 2019, 14, 956-971. [CrossRef] [PubMed]

14. Caetano, A.; Ladeira, F.; Barbosa, R.; Calado, S.; Viana-Baptista, M. Cerebral amyloid angiopathy-The modified Boston criteria in clinical practice. J. Neurol. Sci. 2018, 384, 55-57. [CrossRef]

15. Johnson, K.A.; Gregas, M.; Becker, J.A.; Kinnecom, C.; Salat, D.H.; Moran, E.K.; Smith, E.E.; Rosand, J.; Rentz, D.M.; Klunk, W.E.; et al. Imaging of amyloid burden and distribution in cerebral amyloid angiopathy. Ann. Neurol. 2007, 62, 229-234. [CrossRef]

16. Ly, J.V.; Donnan, G.A.; Villemagne, V.L.; Zavala, J.A.; Ma, H.; O’Keefe, G.; Gong, S.J.; Gunawan, R.M.; Saunder, T.; Ackerman, U.; et al. 11C-PIB binding is increased in patients with cerebral amyloid angiopathy-related hemorrhage. Neurology 2010, 74, 487-493. [CrossRef]

17. Felling, R.J.; Faigle, R.; Ho, C.Y.; Llinas, R.H.; Urrutia, V.C. Cerebral Amyloid Angiopathy: A Hidden Risk for IV Thrombolysis? J. Neurol. Transl. Neurosci. 2014, 2, 1034.

18. Charidimou, A.; Nicoll, J.A.; McCarron, M.O. Thrombolysis-related intracerebral hemorrhage and cerebral amyloid angiopathy: Accumulating evidence. Front. Neurol. 2015, 6, 99. [CrossRef]

19. Davis, J.; Xu, F.; Hatfield, J.; Lee, H.; Hoos, M.D.; Popescu, D.; Crooks, E.; Kim, R.; Smith, S.O.; Robinson, J.K.; et al. A Novel Transgenic Rat Model of Robust Cerebral Microvascular Amyloid with Prominent Vasculopathy. Am. J. Pathol. 2018, 188, 2877-2889. [CrossRef]

20. Verbeek, M.M.; Kremer, B.P.; Rikkert, M.O.; Van Domburg, P.H.; Skehan, M.E.; Greenberg, S.M. Cerebrospinal fluid amyloid beta (40) is decreased in cerebral amyloid angiopathy. Ann. Neurol. 2009, 66, 245-249. [CrossRef]

21. Renard, D.; Castelnovo, G.; Wacongne, A.; Le Floch, A.; Thouvenot, E.; Mas, J.; Gabelle, A.; Labauge, P.; Lehmann, $\mathrm{S}$. Interest of CSF biomarker analysis in possible cerebral amyloid angiopathy cases defined by the modified Boston criteria. J. Neurol. 2012, 259, 2429-2433. [CrossRef] [PubMed]

22. Van Etten, E.S.; Verbeek, M.M.; van der Grond, J.; Zielman, R.; van Rooden, S.; van Zwet, E.W.; van Opstal, A.M.; Haan, J.; Greenberg, S.M.; van Buchem, M.A.; et al. beta-Amyloid in CSF: Biomarker for preclinical cerebral amyloid angiopathy. Neurology 2017, 88, 169-176. [CrossRef] [PubMed]

23. Charidimou, A.; Boulouis, G.; Roongpiboonsopit, D.; Auriel, E.; Pasi, M.; Haley, K.; van Etten, E.S.; Martinez-Ramirez, S.; Ayres, A.; Vashkevich, A.; et al. Cortical superficial siderosis multifocality in cerebral amyloid angiopathy: A prospective study. Neurology 2017, 89, 2128-2135. [CrossRef] [PubMed]

24. Martinez-Ramirez, S.; Pontes-Neto, O.M.; Dumas, A.P.; Auriel, E.; Halpin, A.; Quimby, M.; Gurol, M.E.; Greenberg, S.M.; Viswanathan, A. Topography of dilated perivascular spaces in subjects from a memory clinic cohort. Neurology 2013, 80, 1551-1556. [CrossRef]

25. Brown, R.; Benveniste, H.; Black, S.E.; Charpak, S.; Dichgans, M.; Joutel, A.; Nedergaard, M.; Smith, K.J.; Zlokovic, B.V.; Wardlaw, J.M. Understanding the role of the perivascular space in cerebral small vessel disease. Cardiovasc. Res. 2018, 114, 1462-1473. [CrossRef]

26. Ueno, M.; Chiba, Y.; Murakami, R.; Matsumoto, K.; Fujihara, R.; Uemura, N.; Yanase, K.; Kamada, M. Disturbance of Intracerebral Fluid Clearance and Blood-Brain Barrier in Vascular Cognitive Impairment. Int. J. Mol. Sci. 2019, 20, 2600. [CrossRef]

27. Albargothy, N.J.; Johnston, D.A.; MacGregor-Sharp, M.; Weller, R.O.; Verma, A.; Hawkes, C.A.; Carare, R.O. Convective influx/glymphatic system: Tracers injected into the CSF enter and leave the brain along separate periarterial basement membrane pathways. Acta Neuropathol. 2018, 136, 139-152. [CrossRef]

28. Bakker, E.N.; Bacskai, B.J.; Arbel-Ornath, M.; Aldea, R.; Bedussi, B.; Morris, A.W.; Weller, R.O.; Carare, R.O. Lymphatic Clearance of the Brain: Perivascular, Paravascular and Significance for Neurodegenerative Diseases. Cell Mol. Neurobiol. 2016, 36, 181-194. [CrossRef] 
29. Peng, W.; Achariyar, T.M.; Li, B.; Liao, Y.; Mestre, H.; Hitomi, E.; Regan, S.; Kasper, T.; Peng, S.; Ding, F.; et al. Suppression of glymphatic fluid transport in a mouse model of Alzheimer's disease. Neurobiol. Dis. 2016, 93, 215-225. [CrossRef]

30. Palmqvist, S.; Zetterberg, H.; Blennow, K.; Vestberg, S.; Andreasson, U.; Brooks, D.J.; Owenius, R.; Hagerstrom, D.; Wollmer, P.; Minthon, L.; et al. Accuracy of brain amyloid detection in clinical practice using cerebrospinal fluid beta-amyloid 42: A cross-validation study against amyloid positron emission tomography. JAMA Neurol. 2014, 71, 1282-1289. [CrossRef]

31. Grimmer, T.; Riemenschneider, M.; Forstl, H.; Henriksen, G.; Klunk, W.E.; Mathis, C.A.; Shiga, T.; Wester, H.J.; Kurz, A.; Drzezga, A. Beta amyloid in Alzheimer's disease: Increased deposition in brain is reflected in reduced concentration in cerebrospinal fluid. Biol. Psychiatry 2009, 65, 927-934. [CrossRef] [PubMed]

32. Miebach, L.; Wolfsgruber, S.; Polcher, A.; Peters, O.; Menne, F.; Luther, K.; Incesoy, E.; Priller, J.; Spruth, E.; Altenstein, S.; et al. Which features of subjective cognitive decline are related to amyloid pathology? Findings from the DELCODE study. Alzheimers Res. Ther. 2019, 11, 66. [CrossRef] [PubMed]

33. Li, Q.X.; Villemagne, V.L.; Doecke, J.D.; Rembach, A.; Sarros, S.; Varghese, S.; McGlade, A.; Laughton, K.M.; Pertile, K.K.; Fowler, C.J.; et al. Alzheimer's Disease Normative Cerebrospinal Fluid Biomarkers Validated in PET Amyloid-beta Characterized Subjects from the Australian Imaging, Biomarkers and Lifestyle (AIBL) study. J. Alzheimers Dis. 2015, 48, 175-187. [CrossRef] [PubMed]

34. Catak, C.; Zedde, M.; Malik, R.; Janowitz, D.; Soric, V.; Seegerer, A.; Krebs, A.; During, M.; Opherk, C.; Linn, J.; et al. Decreased CSF Levels of ss-Amyloid in Patients with Cortical Superficial Siderosis. Front. Neurol. 2019, 10, 439. [CrossRef] [PubMed]

35. Sturchler-Pierrat, C.; Abramowski, D.; Duke, M.; Wiederhold, K.H.; Mistl, C.; Rothacher, S.; Ledermann, B.; Burki, K.; Frey, P.; Paganetti, P.A.; et al. Two amyloid precursor protein transgenic mouse models with Alzheimer disease-like pathology. Proc. Natl. Acad. Sci. USA 1997, 94, 13287-13292. [CrossRef]

36. Davis, J.; Xu, F.; Deane, R.; Romanov, G.; Previti, M.L.; Zeigler, K.; Zlokovic, B.V.; Van Nostrand, W.E. Early-onset and robust cerebral microvascular accumulation of amyloid beta-protein in transgenic mice expressing low levels of a vasculotropic Dutch/Iowa mutant form of amyloid beta-protein precursor. J. Biol. Chem. 2004, 279, 20296-20306. [CrossRef]

37. Herzig, M.C.; Winkler, D.T.; Burgermeister, P.; Pfeifer, M.; Kohler, E.; Schmidt, S.D.; Danner, S.; Abramowski, D.; Sturchler-Pierrat, C.; Burki, K.; et al. Abeta is targeted to the vasculature in a mouse model of hereditary cerebral hemorrhage with amyloidosis. Nat. Neurosci. 2004, 7, 954-960. [CrossRef]

38. Herzig, M.C.; Van Nostrand, W.E.; Jucker, M. Mechanism of cerebral beta-amyloid angiopathy: Murine and cellular models. Brain Pathol. 2006, 16, 40-54. [CrossRef]

39. Deane, R.; Bell, R.D.; Sagare, A.; Zlokovic, B.V. Clearance of amyloid-beta peptide across the blood-brain barrier: Implication for therapies in Alzheimer's disease. CNS Neurol. Disord. Drug Targets 2009, 8, 16-30. [CrossRef]

40. Tarasoff-Conway, J.M.; Carare, R.O.; Osorio, R.S.; Glodzik, L.; Butler, T.; Fieremans, E.; Axel, L.; Rusinek, H.; Nicholson, C.; Zlokovic, B.V.; et al. Clearance systems in the brain-implications for Alzheimer disease. Nat. Rev. Neurol. 2015, 11, 457-470. [CrossRef]

41. Ramanathan, A.; Nelson, A.R.; Sagare, A.P.; Zlokovic, B.V. Impaired vascular-mediated clearance of brain amyloid beta in Alzheimer's disease: The role, regulation and restoration of LRP1. Front. Aging Neurosci. 2015, 7, 136. [CrossRef] [PubMed]

42. Wang, W.; Bodles-Brakhop, A.M.; Barger, S.W. A Role for P-Glycoprotein in Clearance of Alzheimer Amyloid beta -Peptide from the Brain. Curr. Alzheimer Res. 2016, 13, 615-620. [CrossRef] [PubMed]

43. Lopez, O.L.; Kuller, L.H.; Mehta, P.D.; Becker, J.T.; Gach, H.M.; Sweet, R.A.; Chang, Y.F.; Tracy, R.; DeKosky, S.T. Plasma amyloid levels and the risk of AD in normal subjects in the Cardiovascular Health Study. Neurology 2008, 70, 1664-1671. [CrossRef] [PubMed]

44. Wood, H. Alzheimer disease: Biomarkers of AD risk-The end of the road for plasma amyloid-beta? Nat. Rev. Neurol. 2016, 12, 613. [CrossRef] [PubMed]

45. Deane, R.; Wu, Z.; Sagare, A.; Davis, J.; Shi, D.Y.; Hamm, K.; Xu, F.; Parisi, M.; LaRue, B.; Hu, H.W.; et al. LRP/amyloid beta-peptide interaction mediates differential brain efflux of Abeta isoforms. Neuron 2004, 43, 333-344. [CrossRef] [PubMed] 
46. Xu, F.; Fu, Z.; Dass, S.; Kotarba, A.E.; Davis, J.; Smith, S.O.; Van Nostrand, W.E. Cerebral vascular amyloid seeds drive amyloid beta-protein fibril assembly with a distinct anti-parallel structure. Nat. Commun. 2016, 7, 13527. [CrossRef]

47. Johnson-Wood, K.; Lee, M.; Motter, R.; Hu, K.; Gordon, G.; Barbour, R.; Khan, K.; Gordon, M.; Tan, H.; Games, D.; et al. Amyloid precursor protein processing and A beta42 deposition in a transgenic mouse model of Alzheimer disease. Proc. Natl. Acad. Sci. USA 1997, 94, 1550-1555. [CrossRef]

48. DeMattos, R.B.; O’Dell, M.A.; Parsadanian, M.; Taylor, J.W.; Harmony, J.A.; Bales, K.R.; Paul, S.M.; Aronow, B.J.; Holtzman, D.M. Clusterin promotes amyloid plaque formation and is critical for neuritic toxicity in a mouse model of Alzheimer's disease. Proc. Natl. Acad. Sci. USA 2002, 99, 10843-10848. [CrossRef]

49. Benveniste, H.; Lee, H.; Ding, F.; Sun, Q.; Al-Bizri, E.; Makaryus, R.; Probst, S.; Nedergaard, M.; Stein, E.A.; $\mathrm{Lu}, \mathrm{H}$. Anesthesia with Dexmedetomidine and Low-dose Isoflurane Increases Solute Transport via the Glymphatic Pathway in Rat Brain When Compared with High-dose Isoflurane. Anesthesiology 2017, 127, 976-988. [CrossRef]

50. Peran, P.; Hagberg, G.; Luccichenti, G.; Cherubini, A.; Brainovich, V.; Celsis, P.; Caltagirone, C.; Sabatini, U. Voxel-based analysis of R2* maps in the healthy human brain. J. Magn. Reson. Imaging 2007, 26, 1413-1420. [CrossRef]

(C) 2020 by the authors. Licensee MDPI, Basel, Switzerland. This article is an open access article distributed under the terms and conditions of the Creative Commons Attribution (CC BY) license (http://creativecommons.org/licenses/by/4.0/). 